\title{
Importance Socio-Économique De Xylopia Aethiopica (Dun) A. Rich. Pour Les Populations Du Sud-Bénin
}

\section{Caroline Ganglo}

Ecole Polytechnique d'Abomey-Calavi, Université d'Abomey-Calavi, Cotonou, Bénin

Laboratoire des Sciences Forestières, Faculté des Sciences Agronomiques, Université d'Abomey-Calavi, Abomey-Calavi, Bénin

\section{Céline Dan}

Ecole Polytechnique d'Abomey-Calavi, Université d'Abomey-Calavi, Cotonou, Bénin

\section{Augustin K. N. Aoudji}

Ecole d'Economie, de Socio-Anthropologie et de Communication pour le développement rural, Cotonou, Bénin

Laboratoire des Sciences Forestières, Faculté des Sciences Agronomiques, Université d'Abomey-Calavi, Abomey-Calavi, Bénin

\section{Alain Jaures Gbetoho}

Laboratoire des Sciences Forestières, Faculté des Sciences Agronomiques,

Université d'Abomey-Calavi, Abomey-Calavi, Bénin

\section{Jean C. Ganglo}

Laboratoire des Sciences Forestières, Faculté des Sciences Agronomiques, Université d'Abomey-Calavi, Abomey-Calavi, Bénin

Doi: 10.19044/esj.2017.v13n33p187 URL:http://dx.doi.org/10.19044/esj.2017.v13n33p187

Abstract

Xylopia aethiopica is a multi-purpose Non Timber Forest Product (NTFP) which products are consumed across Africa. This study aimed at generating useful information to support the sustainable management of Xylopia aethiopica in southern Benin. A survey was carried out to interview 122 stakeholders, including consumers and traders. Data were collected on the consumption of Xylopia aethiopica products, the income generated by their marketing, and the management the species trees by local people. As results, the species was mainly used in traditional medicine. Other forms of consumption included: spiritual rituals, fuelwood, food, and construction timber. Among Xylopia aethiopica products, the fruits were the most commercialized. The stakeholders operating in the commercialization system were: collectors, processors-wholesalers, and retailers. The gross margin per $100 \mathrm{~kg}$ of fruits averaged XOF 4500 for collectors and processors- 
wholesalers, and XOF 2500 for retailers. Xylopia aethiopica trees were privately managed based on land ownership. Given the decrease of the species, because of over-exploitation and cultural beliefs, the sensitizing of local people and the promotion of domestication are expected to support its conservation.

Keywords: Xylopia aethiopica, Non-Timber Forest Product, marketing, income, Benin

\section{Résumé}

Xylopia aethiopica est une espèce de Produit Forestier Non Ligneux (PFNL) utilisée à diverses fins par les populations africaines. La présente étude a pour objectif de générer les informations permettant une gestion durable de Xylopia aethiopica au Sud-Bénin. Une enquête a été faite auprès de 122 acteurs composés de consommateurs et de commerçants des produits de Xylopia aethiopica. Des données ont été collectées sur les formes d'utilisation de l'espèce, les retombées financières de la commercialisation de ses organes, et les modes de gestion par les populations. Comme résultats, les usages médicinaux de Xylopia aethiopica sont les plus fréquents, suivis des usages magico-religieux, bois énergie, ressource alimentaire et bois de construction. Le fruit est 1'organe de Xylopia aethiopica le plus commercialisé. Les principaux acteurs impliqués dans le système de commercialisation des fruits sont: les collecteurs, les transformatricesgrossistes et les détaillants. La marge brute moyenne par sac de $100 \mathrm{~kg}$ est de 4500 FCFA pour les collecteurs et les transformatrices-grossistes, et 2500 FCFA pour les détaillants. L'espèce fait l'objet d'une gestion privée, avec une propriété associée à celle de la terre. Une régression de Xylopia aethiopica a été observée dans la zone d'étude, à cause de sa sur-exploitation et des croyances culturelles. La sensibilisation des populations et la promotion de la domestication contribueront à la conservation de l'espèce.

Mots-Clés: Xylopia aethiopica, Produits Forestiers Non Ligneux, commercialisation, revenus, Bénin

\section{Introduction}

La place de la diversification des moyens d'existence dans les stratégies paysannes a été largement reconnue au cours des dernières années, notamment en Afrique au Sud du Sahara (Bigsten et Tengstam, 2011 ; Hilson, 2011). Dans cette perspective, les Produits Forestiers Non Ligneux (PFNL) contribuent à l'économie des ménages en générant des revenus, et en fournissant divers produits, d'où leur fonction essentielle, notamment en temps de soudure (Fidele et al., 2010 ; Vantomme et Gazza, 2010 ; Zanh et 
al., 2016). Le terme « Produits Forestiers Non Ligneux » désigne l'ensemble des produits (autres que le bois d'œuvre et d'industrie ainsi que leurs dérivés) qui sont extraits d'écosystèmes forestiers et qui sont utilisés à des fins domestiques ou de commercialisation ou dotés d'une signification sociale, culturelle ou religieuse (FAO, 2001). Ainsi définis, les PFNL comprennent les produits tirés des forêts, des autres terres boisées et des arbres hors forêt (FAO, 2009).

De nombreuses études (Codjia et al., 2003, Poissonnet et al., 2006; Rouxel, 2010) révèlent que les PFNL ont le potentiel socioéconomique de contribuer au développement durable. En effet, les produits commercialisés contribuent à satisfaire des besoins quotidiens et assurent des emplois ainsi que des revenus, pour les populations rurales, en particulier les femmes (Awono et al., 2008 ; Manirakiza, 2007). Les populations en dépendent comme sources d'aliments, de fourrage, de médicaments, de résine, de matériaux de construction, etc. (Brown, 2005). Il convient cependant de notifier que depuis quelques années, les produits forestiers non ligneux connaissent un regain d'intérêt, ce que l'Organisation des Nations Unies attribue à « une prise de conscience accrue de leur contribution à l'économie des ménages et à la sécurité alimentaire, à quelques économies nationales, et à certains objectifs écologiques, tels que la conservation de la diversité biologique »(FAO, 1999). En effet, l'importance socio-économique d'une espèce peut être un atout en faveur de sa promotion (Moupela et al., 2011) et la connaissance de sa gestion actuelle peut guider le choix de méthodes appropriées de gestion.

Xylopia aethiopica (Dun) A. Rich., est une angiosperme de la famille des Annonaceae, connue en Afrique pour les PFNL qu'elle procure aux populations locales (Abaidoo et al., 2011). Compte tenu de l'importance de l'espèce pour les communautés rurales, il importe d'élargir et d'approfondir les connaissances afin éclairer les décideurs sur la pertinence des actions de conservation. Ainsi, cet article traite des trois questions suivantes : quelle est la diversité des utilisations de Xylopia aethiopica? Quels sont les retombées financières de la commercialisation de ses organes ? Quel est le mode de gestion actuel de ces peuplements ? Ces questions fondent la présente étude dont l'objectif est d'évaluer l'importance socio-économique de Xylopia aethiopica pour les populations du Sud-Bénin.

\section{Méthodes}

\section{Collecte de données}

L'étude a été conduite au Sud-Bénin dans les communes d'AbomeyCalavi (département de l'Atlantique), Cotonou (département du Littoral), Avrankou et Adjara (département de l'Ouémé) (Figure 1). Les communes d'Avrankou et Adjara ont été sélectionnées parce qu'elles abritent des 
peuplements de Xylopia aethiopica, tandis qu'Abomey-Calavi et Cotonou ont été choisies à cause de la commercialisation des produits de Xylopia aethiopica sur leurs marchés.

Une enquête a été menée auprès des différents acteurs composés de commerçants et des utilisateurs des organes de Xylopia aethiopica. A cet effet, un échantillonnage stratifié a été fait en fonction des localités (premier niveau) et des acteurs (deuxième niveau). Cela a permis de sélectionner 122 acteurs dont 50 commerçants et 72 utilisateurs des produits de Xylopia aethiopica. Les données ont été collectées auprès de chaque type d'acteur grâce à des entretiens structurés à base d'un questionnaire. Outre les caractéristiques sociodémographiques de l'enquêté, les informations collectées concernent: les formes d'utilisation des organes de Xylopia aethiopica, les retombées financières de la commercialisation de ces organes, et le mode de gestion des pieds de l'espèce.

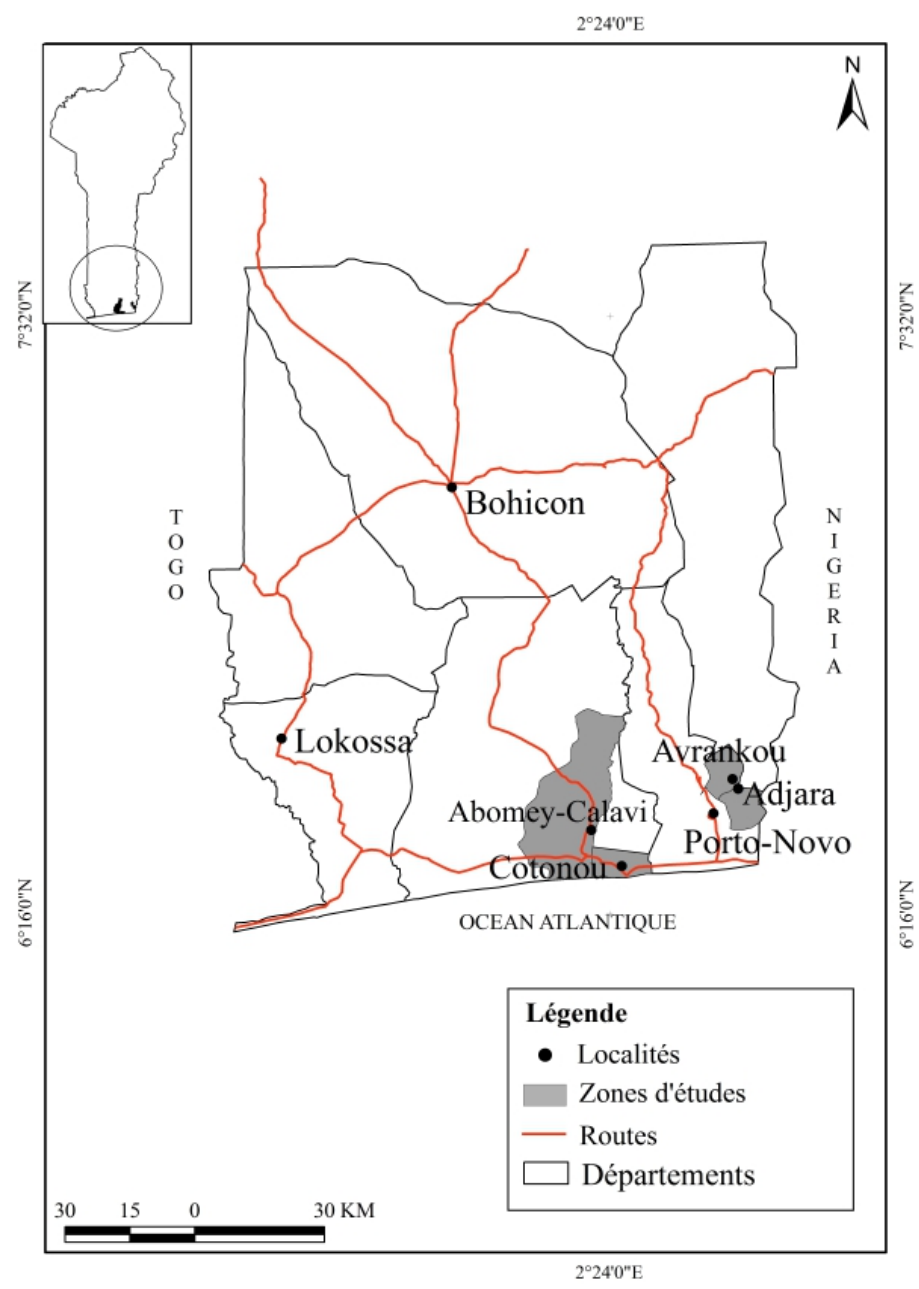

Figure 1 : Carte du Bénin indiquant les différentes zones d'étude. 


\section{Traitement et analyse des données}

Le traitement des données comprend trois points: (i) les formes d'utilisation des organes de Xylopia aethiopica, (ii) les retombées financières de la commercialisation des organes, et (iii) le mode de gestion de l'espèce par la population.

Les formes d'utilisation des organes de Xylopia aethiopica ont été décrites sur la base des fréquences relatives calculées comme suit : $\boldsymbol{F}_{\boldsymbol{i}}=\boldsymbol{n}_{\boldsymbol{i}}$ $\times \mathbf{1 0 0} / \boldsymbol{N}$; avec $\boldsymbol{F}_{\boldsymbol{i}}$, la fréquence relative pour une utilisation $i ; \boldsymbol{n}_{\boldsymbol{i}}$, le nombre de personnes ayant fourni une réponse positive pour une utilisation donnée ; et $N$, le nombre de répondants.

Les fruits étant l'organe de Xylopia aethiopica le plus échangé sur le marché, les retombées financières de leur commercialisation ont été évaluées à travers la marge brute. Pour chaque type d'acteur, les charges variables, les recettes et la marge brute ont été calculées. Ces divers paramètres ont été déterminés pour un sac de $100 \mathrm{~kg}$ de fruits, afin de permettre les comparaisons entre acteurs. La marge brute a été calculée comme suit (Desbois, 2006) :

\section{Marge brute $=$ Prix de vente $\boldsymbol{-}$ charges variables}

L'analyse du mode de gestion de Xylopia aethiopica par les populations a porté sur les points suivants : l'origine des peuplements, les techniques de collecte des organes, les soins apportés à l'espèce et la perception sur l'évolution de la ressource.

\section{Résultats}

\section{Formes d'utilisation des organes de Xylopia aethiopica}

Cinq formes d'usage des organes de Xylopia aethiopica ont été recensées (figure 2) : les usages médicinaux, alimentaires, magico-réligieux, bois énergie et bois de construction. Les usages médicinaux sont les plus fréquents (plus de $60 \%$ ) suivis des usages magico-religieux $(22 \%)$. Les usages comme bois de chauffe, ressource alimentaire et bois de construction sont les moins fréquents $(18 \%)$.

Les racines, les fruits, les feuilles et l'écorce sont les organes de Xylopia aethiopica utilisés à des fins médicinales (Figure 3). Les fruits sont plus utilisés ( $50 \%$ des enquêtés). La racine, l'écorce et les feuilles sont moins utilisées.

En ce qui concerne les usages magico-religieux des organes de Xylopia aethiopica, les fruits sont utilisés pour les paris et lors des cérémonies funéraires. Les fruits, les feuilles et les racines sont utilisés pendant les cérémonies de purification de l'homme ou de la femme qui éprouve des difficultés à fonder un foyer. Par ailleurs, l'espèce constitue un arbre sacré, et est planté dans les couvents. 


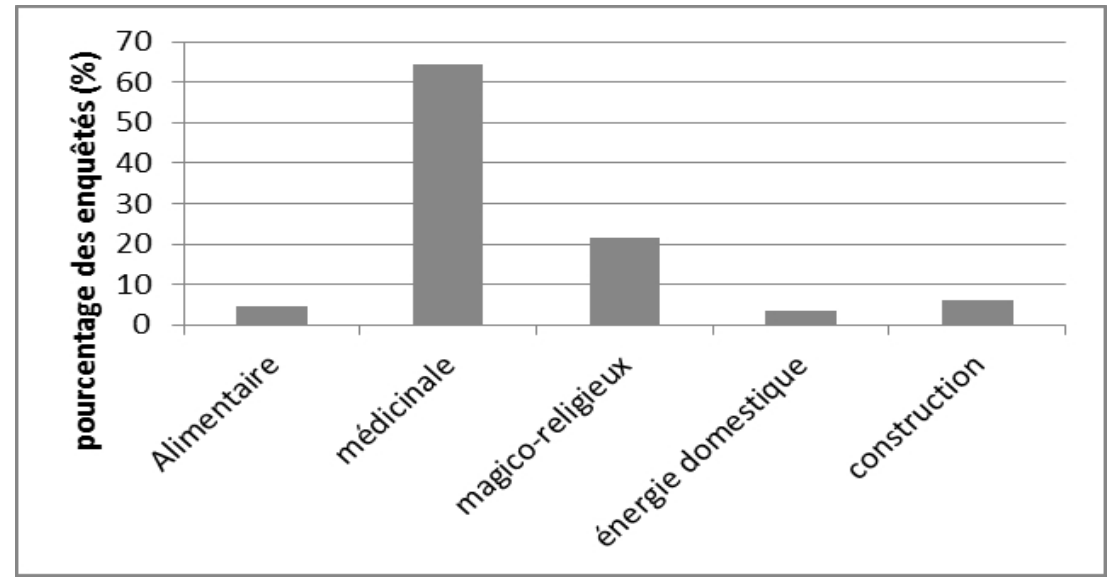

Figure 2: Formes d'utilisation des organes de Xylopia aethiopica.

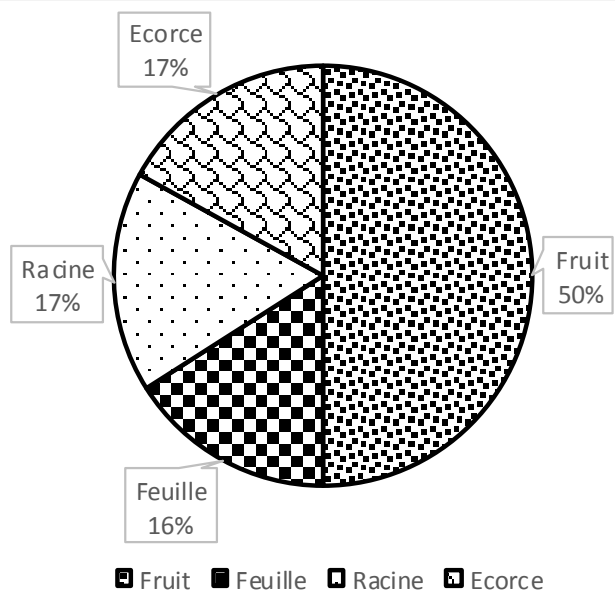

Figure 3 : Fréquence d'utilisation des organes de Xylopia aethiopica à des fins médicinales.

Retombées financières de la commercialisation des organes de Xylopia aethiopica

Acteurs impliqués dans la commercialisation des organes de Xylopia aethiopica

Les organes de Xylopia aethiopica commercialisés sont les fruits, les racines et l'écorce. Toutefois, les racines et écorces ne font l'objet que d'échanges limitées. Ainsi, la description des fonctions des acteurs dans le système de commercialisation concerne le fruit qui est de loin l'organe le plus échangé. Les principaux acteurs du système de commercialisation des fruits de Xylopia aethiopica sont: les collecteurs, les transformatricesgrossistes et les détaillants.

Les collecteurs constituent le premier maillon de la chaîne de commercialisation des fruits de Xylopia aethiopica. Ils sont basés dans les communes d'Adjara et Avrankou (Figure 1). Ce sont des hommes dont l'âge 
est compris entre 30 et 50 ans. Les fruits sont collectés pendant la saison sèche, et sont ensuite conditionnés dans les sacs de $100 \mathrm{~kg}$ pour être livrés aux commerçants (Figure 4).

Les transformatrices-grossistes achètent les fruits auprès des collecteurs. Elles procèdent à la transformation qui consiste à fumer les fruits verts, afin de les noircir (figure 5). Cette opération permet d'accroître la valeur du produit et de le conserver durant toute la période de vente. Les transformatrices-grossistes sont des femmes qui opèrent dans les marchés d'Adjara et d'Avrankou.

Les détaillants achètent les fruits noirs de Xylopia aethiopica auprès des transformatrices-grossistes dans les marchés d'Adjara (52\%), d'Avrankou (36\%) et de Dantokpa (Cotonou) (12\%). C'est auprès d'eux que les consommateurs s'approvisionnent dans les différents marchés. Les fruits noirs de Xylopia aethiopica sont vendus en détail sous forme de petits tas (figure 5). Les détaillants sont en grande majorité des femmes (91\%), âgées de 25 à 70 ans. Leurs activités concernent la commercialisation des plantes médicinales en général. Les hommes $(9 \%)$ sont âgés de 40 à 60 ans et exercent aussi la profession de guérisseur traditionnel.

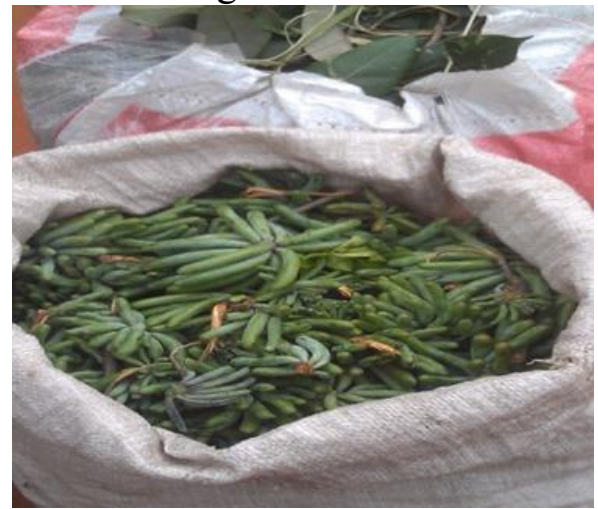

Figure 4 : Sac de fruits verts de Xylopia aethiopica vendu à 6000 FCFA.

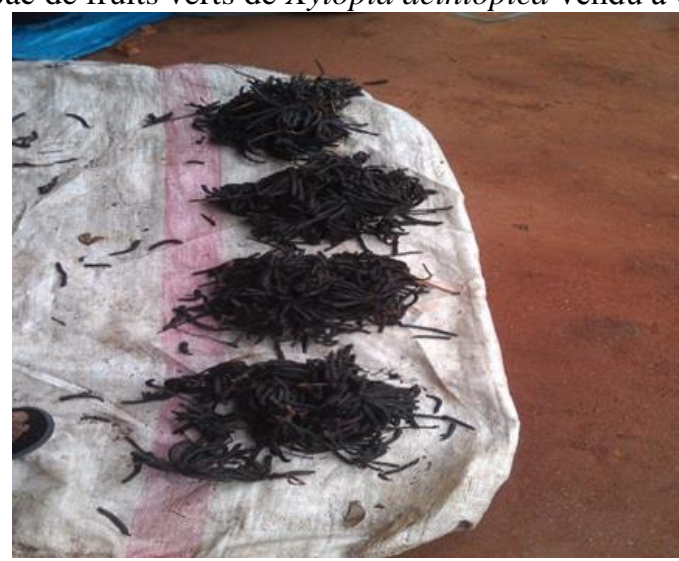

Figure 5 : Tas de fruits noirs de Xylopia aethiopica vendu à 500 FCFA l'unité. 


\section{Coûts et marges de commercialisation des fruits de Xylopia aethiopica}

Les coûts variables de l'exploitation d'un sac de $100 \mathrm{~kg}$ de fruit de Xylopia aethiopica varient selon les acteurs (Tableau 1). Au niveau des collecteurs, les coûts variables (1500 FCFA/sac de $100 \mathrm{~kg}$ ) regroupent les frais de main-d'œuvre occasionnelle, le transport et l'achat de sacs. Au niveau des transformatrices-grossistes, les charges supportées sont de 7500 FCFA/sac de $100 \mathrm{~kg}$ en moyenne, et sont liées à l'achat des fruits verts, le transport et l'achat du bois de chauffe. Chez les détaillants, les coûts variables (12500 FCFA/sac de $100 \mathrm{~kg}$ ) comprennent l'achat de fruits noirs (transformés) et des sachets pour le conditionnement.

Tableau 1 : Coûts variables pour l'exploitation d'un sac de $100 \mathrm{~kg}$ de fruits de Xylopia aethiopica en fonction des acteurs (en FCFA).

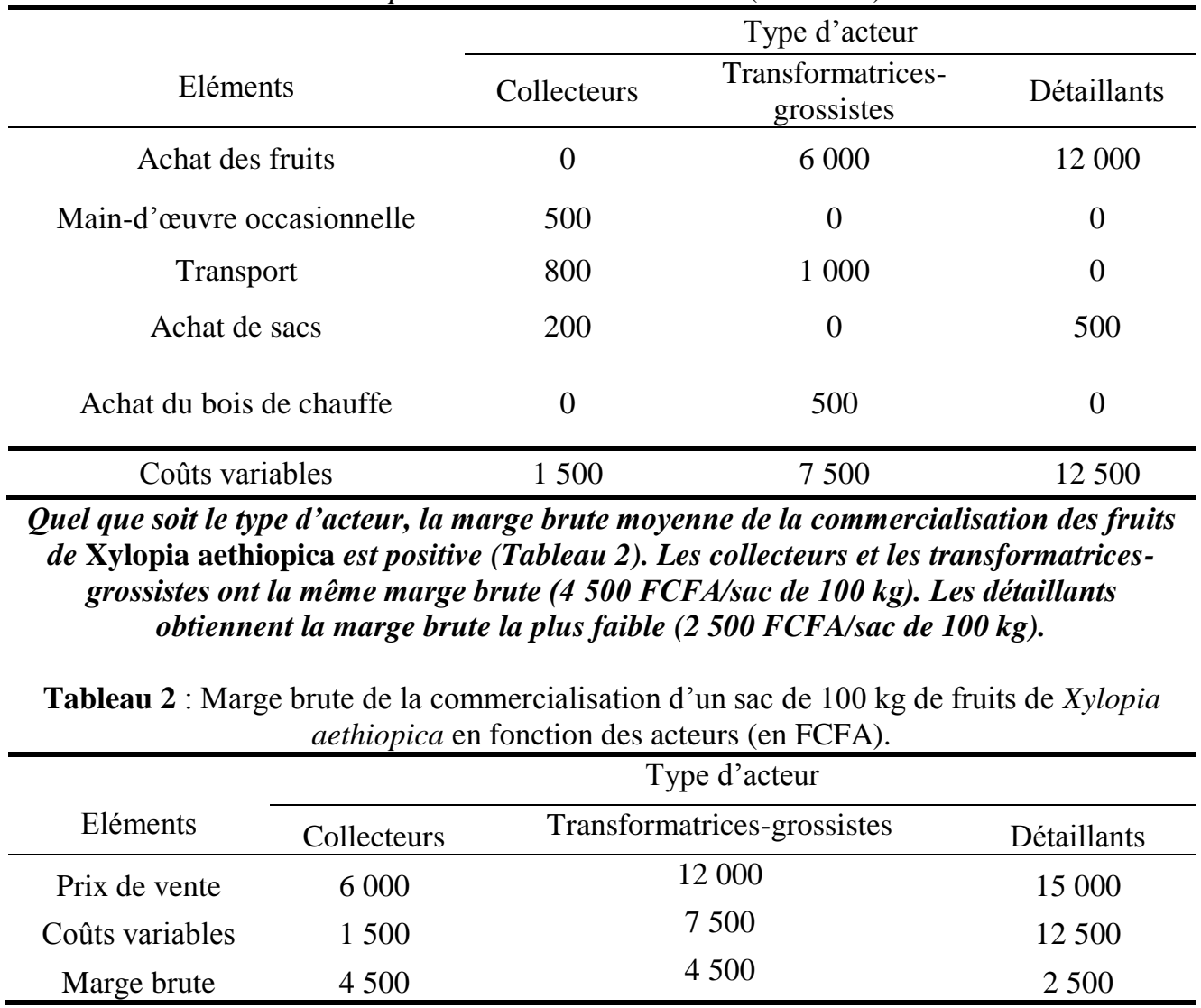

\section{Gestion des pieds de Xylopia aethiopica}

Les propriétaires des pieds de Xylopia aethiopica sont des hommes. Ils constituent aussi les collecteurs dans la filière de commercialisation des organes de l'espèce. La propriété des pieds de Xylopia aethiopica est liée à celle de la terre qui abrite les arbres. Ainsi, il existe des cas de transmission par héritage d'une génération à l'autre. 
Les pieds de Xylopia aethiopica proviennent des peuplements naturels. En effet, aucun propriétaire enquêté n'a rapporté avoir planté les pieds qu'il exploite. Le nombre de pieds de Xylopia aethiopica par propriétaire varie entre 4 à 16 , avec une moyenne de 8 pieds.

Généralement, les pieds de Xylopia aethiopica ne sont pas entretenus par les propriétaires. Dans quelques cas, le désherbage est effectué pour libérer l'espèce de la concurrence des adventices. Cette opération facilite également l'accès aux arbres pour la récolte des organes.

En ce qui concerne la technique d'exploitation des organes, les feuilles sont obtenues suite à l'abattage des arbres ou la coupe des branches. La récolte des fruits se fait en secouant l'arbre, ou en utilisant un long bois pour les faire tomber. Les écorces sont coupées à l'aide d'une machette et les racines sont obtenues après l'abattage des arbres. Ces actions contribuent à la destruction de l'espèce.

Les populations ont rapporté une tendance régressive des peuplements de Xylopia aethiopica dans le milieu (décroissance du nombre de pieds au fil des années). En considérant la période des vingt dernières années, les individus de Xylopia aethiopica auraient diminué de moitié dans le milieu d'étude. Deux grands facteurs sont avancés par les populations pour expliquer cette tendance régressive : les pressions anthropiques sur l'espèce et les facteurs socioculturels.

\section{Discussion}

\section{Formes d'utilisation des organes de Xylopia aethiopica}

Plusieurs utilisations des organes de Xylopia aethiopica ont été recensées : usages médicinal, alimentaire, magico-religieux, bois énergie et bois de construction. D'autres travaux conduits sur les PFNL ont rapporté des formes d'utilisation similaires à celles rencontrées dans la zone d'étude (Assogbadjo, 2000 ; Orwa et al., 2009 ; Zinsouklan et al., 2014 ; Zanh et al., 2016). Ces diverses utilisations démontrent l'importance socioculturelle de Xylopia aethiopica dans la vie des populations.

L'utilisation de Xylopia aethiopica à des fins médicinales est plus fréquente dans la zone d'étude. Ce résultat corrobore les données fournies par l'OMS (2011) selon laquelle plus de $80 \%$ des populations africaines ont recours aux plantes médicinales pour se soigner, du fait de leur accessibilité géographique, économique et culturelle facile. En effet, toutes les parties de Xylopia aethiopica sont très utiles en médecine traditionnelle, mais les fruits sont les plus utilisés; ce qui traduit leur importance en médecine traditionnelle (Fetse et al., 2016 ; Orwa et al., 2009).

L'utilisation du bois de Xylopia aethiopica dans la construction entraîne sa destruction. En effet, l'espèce est recherchée pour la rectitude de 
son bois et sa résistance contre les attaques des insectes. Il est donc nécessaire d'œuvrer au renouvellement de Xylopia aethiopica dans le milieu.

\section{Retombées financières de la commercialisation des organes de Xylopia aethiopica}

Le fruit constitue l'organe de Xylopia aethiopica le plus commercialisé. Trois principaux acteurs animent le système de commercialisation de ce produit: les collecteurs, les transformatricesgrossistes et les détaillants. La description des fonctions de ces acteurs montre que la chaîne de commercialisation des fruits de Xylopia aethiopica est courte et peu complexe. Les échanges se font seulement sur les marchés locaux, par opposition à Voacanga africana au Bénin dont le circuit de commercialisation comporte d'autres intermédiaires (tels que les courtiers), en plus des acteurs traditionnels (exploitants, grossistes, détaillants, consommateurs) (Fafeh, 2015).

Pour l'exploitation d'un sac de $100 \mathrm{~kg}$ de fruits de Xylopia aethiopica, les coûts d'opération sont plus élevés au niveau des transformatrices-grossistes et des détaillants, par rapport aux collecteurs. Les coûts plus faibles des collecteurs sont dus au fait qu'ils sont les propriétaires des pieds de Xylopia aethiopica, et n'achètent donc pas les fruits.

La marge brute moyenne générée par l'exploitation d'un sac de 100 $\mathrm{kg}$ de fruit de Xylopia aethiopica est positive pour tous les acteurs. Ainsi, la commercialisation des fruits de Xylopia aethiopica contribue aux revenus des populations. Cette étude confirme que les Produits Forestiers Non Ligneux (PFNLs) sont des sources de revenus pour les acteurs du système de commercialisation (Guédjé, 2002 ; Akpovi, 2011).

Les collecteurs perçoivent la même marge brute que les transformatrices-grossistes; les détaillant se classent derniers. En effet, les charges au niveau des collecteurs n'incluent pas l'achat des fruits de Xylopia aethiopica, ce qui contribue à accroître leur marge brute. Quant aux transformatrices-grossistes, elles ajoutent de la valeur aux fruits verts achetés chez les collecteurs, ce qui augmente leur marge brute par rapport à celle des détaillants.

La collecte et la commercialisation des fruits frais de Xylopia aethiopica se font pendant la saison sèche au Sud-Bénin, période à laquelle les fruits arrivent à maturité. Il s'agit le plus souvent d'un commerce ponctuel pour les propriétaires des pieds; et les revenus tirés de leurs ventes permettent l'acquisition d'autres produits alimentaires et non alimentaires nécessaires aux ménages (Manirakiza, 2007 ; Lescuyer, 2010). 


\section{Gestion des pieds de Xylopia aethiopica}

La prépondérance des hommes parmi les propriétaires des pieds de Xylopia aethiopica est due au fait que la possession de pieds est intimement liée à la propriété sur la terre. En effet, en Afrique, et au Bénin en particulier, les femmes ont un accès limité à la terre (Thiessen, 2016; World Bank, 2011). Il existe des disparités dans le nombre de pieds possédés par les propriétaires. Ces disparités seraient liées à l'étendue du patrimoine foncier des propriétaires.

La collecte des organes de Xylopia aethiopica par abattage ne permet pas la régénération de l'espèce. Cette méthode d'exploitation n'est pas durable (Tiorambe, 2007). En effet, l'abattage de Xylopia aethiopica pour la collecte des organes peut à terme affecter la capacité de régénération naturelle de l'espèce et la viabilité de ses peuplements.

Il a été observé une tendance de régression des peuplements de Xylopia aethiopica dans la zone d'étude. Ce résultat confirme celui de Akouehou et al. (2016) au Bénin qui montrent que Xylopia aethiopica est une espèce rare, avec un indice de rareté de $89 \%$ (le seuil de rareté étant 80\%). Neuenschwander et al. (2011) avaient aussi rapporté que les fruits de Xylopia aethiopica sont fortement exploités au point que la survie de l'espèce est menacée. Cette régression de l'espèce est due aux facteurs anthropiques et culturels. Les pressions anthropiques se traduisent par l'abattage des pieds de Xylopia aethiopica pour la construction des habitats (implantations humaines), le prélèvement incontrôlé des organes, la coupe abusive du bois pour divers usages. L'intensité de l'exploitation des organes de l'espèce est due au degré de commercialisation du produit. Il a été démontré que l'accès au marché ou la proximité du marché ont des conséquences écologiques sur la ressource (Shanley et al., 2002). Plus la demande d'un PFNL est élevée, plus la pression sur la ressource est forte.

Les facteurs socioculturels affectent aussi Xylopia aethiopica. En effet, l'espèce serait un refuge des hiboux qui sont considérés au Bénin comme des animaux de compagnie des sorciers. Cette croyance répandue est à l'origine de la destruction des pieds de Xylopia aethiopica dans les concessions.

Comme palliatif à la régression de Xylopia aethiopica, la domestication a été la solution proposée à la suite d'autres chercheurs ayant étudié les PFNL (Ræbild et al., 2011 ; Zinsouklan et al., 2014). En effet, la domestication joue un rôle important dans l'amélioration, la gestion et la valorisation des ressources naturelles, et l'augmentation des revenus des populations rurales (Schreckenberg et al., 2006). A la domestication, il faudrait associer des campagnes de sensibilisation, afin de faire évoluer la croyance répandue selon laquelle les pieds de Xylopia aethiopica sont des refuges des hiboux et porteraient malchance au propriétaire. 


\section{Conclusion}

La présente étude conduite au Sud-Bénin a permis d'évaluer les utilisations de Xylopia aethiopica par les populations et son potentiel économique pour une gestion durable de l'espèce. Les organes utilisés de l'espèce sont : les fruits, les feuilles, les racines et l'écorce. Ces organes sont employés sous diverses formes à savoir : les usages médicinaux qui sont les plus fréquents, les usages alimentaires, les usages magico-religieux, les utilisations comme bois de chauffe et bois de construction.

Le fruit de Xylopia aethiopica est l'organe le plus échangé sur le marché; et sa commercialisation génère des marges brutes positives aux différents acteurs que sont : les collecteurs, les transformatrices-grossistes et les détaillants.

La propriété des pieds de Xylopia aethiopica au Sud-Bénin est liée à la propriété de la terre qui abrite le peuplement. Les pressions anthropiques sur l'espèce et les facteurs socioculturels sont à la base de sa régression au Sud-Bénin.

Au regard de l'intérêt reconnu des organes de Xylopia aethiopica, de leur apport substantiel dans les revenus des ménages, une attention devrait être accordée à sa promotion par les décideurs. Etant donné que les activités de commercialisation ont lieu pendant la période de soudure, Xylopia aethiopica, à l'instar d'autres PFNL, pourrait être considérée dans les stratégies d'amélioration des moyens d'existence des populations. Pour garantir la disponibilité de la ressource, il est nécessaire de promouvoir sa domestication. Il est également important de sensibiliser la population sur les méthodes d'exploitation qui ne menacent pas la survie de l'espèce.

\section{References:}

1. Akouehou, G., Legba, S. I. \& Orou, M. A. (2016). Chaîne de valeur de quelques plantes médicinales utilisées et connaissances traditionnelles associées dans quelques centres urbains au Bénin. 60 p.

2. Akpovi, R. (2011). Commercialisation et valorisation des feuilles de teck dans le département de l'Atlantique (Sud-Bénin). Mémoire de maitrise, Département de Géographie et Aménagement du Territoire. FLASH/UAC, pp. 61-67.

3. Assogbadjo, A. E. (2000). Etude de la biodiversité des ressources forestières alimentaires et évaluation de leur contribution à l'alimentation des populations locales de la forêt classée de la Lama. Thèse d'ingénieur agronome, FSA-UNB, 131p.

4. Awono, A., Manirakiza, D. \& Owona, H. (2008). Etude de base de la filière miel, Gnetum spp et Dacryodes edulis dans les provinces du Bas Congo et de Kinshasa (RDC). GCP/RAF/408/EC, « Mobilisation 
et Renforcement des capacités des petites et moyennes entreprises impliquées dans les filières des produits forestiers non ligneux en Afrique Centrale », CIFOR.

5. Bigsten, A. \& Tengstam, S. (2011). Smallholder Diversifcation and Income Growth in Zambia. Journal of African Economies, 20, 781822.

6. Brown, H. C. P. (2005). Governance of Non-Wood Forest Products And Community Forests In The Humid Forest Zone Of Cameroon. PhD Dissertation. Cornell University.

7. Codjia, J. T. C., Assogbadjo, A. E. \& Mensag, M. R. (2003). Diversité et valorization au niveau local des ressources forestières alimentaires du Bénin. Cahier Agricultures, 12, 321-331.

8. Desbois, D. (2006). Méthodologie d'estimation des coûts de production agricole : comparaison de deux méthodes sur la base du Rica. Revue modulad, 35, 45-72.

9. Fafeh, A. E. K. (2015). Produits Forestiers Non Ligneux au Bénin : Commercialisation des graines de Voacanga africana et implications pour sa conservation. Editions universitaires européennes. 68p.

10. FAO (1999). La FAO et la foresterie : Vers une définition harmonisée des produits forestiers non ligneux. Unasylva, 198, 6364.

11. FAO (2001). Evaluation des produits forestiers ligneux au Sénégal. FAO, Bureau régional de l'Afrique, Accra, Ghana, 2 ème édition.

12. FAO. (2009). " L'amélioration des revenus et de la sécurité alimentaire des petits exploitants en Afrique de l'Ouest et en Afrique Centrale par l'exportation de produits tropicaux biologiques et $d u$ commerce équitable »: Evaluation d'impact du projet Beurre de karité au Burkina Faso. Rome.

13. Fetse, J. P., Kofie, W. \& Adosraku, R. K. (2016). Ethnopharmacological Importance of Xylopia aethiopica (DUNAL) A. RICH (Annonaceae) - A Review. British Journal of Pharmaceutical Research, 11(1), 1-21.

14. Fidele, G., Urech, Z. L., Rehnus, M. \& Sorg, J. P. (2010). Impact of women's harvest practices on Pandanus guillaumetii in Madagascar's lowland rainforests. Econ. Bot., 65, 158-168.

15. Guédjé, N. M. (2002). La gestion des populations d'arbres comme outil pour une exploitation durable des Produits Forestiers NonLigneux : l'exemple de Garcinia lucida (Sud-Cameroun). Thèse de Doctorat. Univ. Lib. Bruxelles. Belgique, 206 p.

16. Hilson, G. (2011). Artisanal mining, smallholder farming and livelihood diversification in rural sub-Saharan Africa: an introduction. Journal of International Development, 23, 1031-1041. 
17. Lescuyer, G. (2010). Importance économique des produits forestiers non ligneux dans quelques villages du sud-Cameroun. Bois Forêts Trop, 304, 15-24.

18. Manirakiza, D. (2007). Etude de la consommation d'Irvingia spp. (Mangue Sauvage) et Ricinodendron heudelotii (Njansang) à Yaoundé et Libreville.

19. Moupela, C., Vermeulen, C., Daïnou, K. \& Doucet, J.-L. (2011). Le noisetier d'Afrique (Coula edulis Baill.). Un produit forestier non ligneux méconnu. Biotechnol. Agron. Soc. Environ, 15, 451-461.

20. Neuenschwander, P., Sinsin, B. \& Goergen, G. (2011). Protection de la Nature en Afrique de l'Ouest: Une Liste Rouge pour le Bénin. Nature Conservation in West Africa: Red List for Benin. International Institute of Tropical Agriculture, Ibadan, Nigeria. 365 p.

21. OMS (2011). Rapport narratif : profil pharmaceutique de la République démocratique du Congo 2011. Kinshasa, RDC, Ministère de la Santé publique, $32 \mathrm{p}$.

22. Orwa, C., Mutua, A., Kindt, R., Jamnadass, R. \& Anthony, S. (2009). Agroforestree Database: a tree reference and selection guide version 4.0. World Agroforestry Centre, Kenya.

23. Poissonnet, M., Parmantier, A., Wyngaarde, B., Demenois, J. \& Lescuyer, G. (2006). Espoirs et difficultés du transfert de la gestion forestière à deux communautés de Guyane et du Cameroun. Bois et forêts des tropiques, 289(3), 5-16.

24. Raebild A., Larsen A. S., Jensen J. S., Ouedrago M., De Groote S., Van Damme P., Bayala J., Diallo B.O., Sanou H., Kalinganire A. \& Kjaer E.D. (2011). Advances in domestication of indigenous fruit trees in the West African Sahel. New Forest, 41, 297-315.

25. Rouxel, C. (2010). Conservation de la biodiversité et développement durable des territoires. Économie rurale, 6, 39-52.

26. Schreckenberg, K., Awono, A., Degrande, A., Mbosso, C., Ndoye, O. \& Tchoundjeu, Z. (2006). Domesticating indigenous fruit trees as a contribution to poverty reduction. Forests, Trees and Livelihoods, 16, $35-51$.

27. Shanley, P., Pierce, A. R., Laird, S. A. \& Guillén, A. (2002). Tapping the Green Market: Certification and Management of Non-Timber Forest Products. Earthscan, London.

28. Thiessen, C. (2016). Equal harvests: How investing in agricultural development can empower women. Canadian Foodgrains Bank, 28p.

29. Tiorambe, B. (2007). Valorisation des PFNL de Valorisation des PFNL de la RDC : atout et rôle futur dans la gestion des forêts. 
30. Vantomme, P. \& Gazza, S. (2010). Le défi de la sylviculture en faveur des produits forestiers non ligneux sous les tropiques : de la cueillette à l'agriculture ? Bois Forêts Trop, 304, 5-13.

31. Abaidoo, C. S., Woode, E., Chrissie, S., \& Alhassan, A. (2011). An evaluation of the effect of ethanolic fruit extracts of Xylopia aethiopica on haematological and biochemical parameters in male rats. Der Pharmacia Sinica, 2 (2), 39-45.

32. World Bank (2011). World Development Report 2012: Gender equality and development. Washington, D.C.: World Bank.

33. Zanh, G. G., Barima, Y. S. S., Kouakou, K. A. \& Sangne, Y. C. (2016). Usages des produits forestiers non-ligneux selon les communautés riveraines de la forêt classée du Haut-Sassandra (Centre-Ouest de la Côte d'Ivoire). Int. J. Pure App. Biosci, 4(5), 212-225.

34. Zinsouklan, J., Toyi, M., Aoudji, A., Tenté, B., Hounnou, F. \& Houinato, M. (2014). Vers une valorisation durable des espèces negligées : formes d'utilisations, retombées financières et modes de gestion de Parkia biglobosa (jacq.) R.br. Ex Benth. Au centre bénin. Science de la vie, de la terre et agronomie, 2 (2), 21-27. 